\title{
Analysis and Experimental Demonstration of Simplex Coding Technique for SNR Enhancement of OTDR
}

\author{
Duckey Lee, Hosung Yoon, Na Young Kim, Hansuek Lee, and Namkyoo Park \\ Optical Communication Systems Laboratory, School of Electrical Engineering and Computer \\ Sciences, Seoul National University, Seoul, 151-742, Korea \\ Tel: +82-2-880-1820, Fax: +82-2-885-5284, E-mail: duckey@ieee.org
}

\begin{abstract}
We demonstrate SNR improvement of OTDR using simplex codes by experiment, for the first time. For this, we proposed a practical algorithm and derived detailed equations. An in-house OTDR board was developed to verify the proposed algorithm, and the experimental results showed excellent agreement with theory. In addition, we analyzed the SNR variation of the simplex code OTDR, caused by bandlimited receiver characteristics.
\end{abstract}

Index Terms - Optical fiber communications, Optical time domain reflectometry (OTDR), Simplex codes.

\section{INTRODUCTION}

The optical time domain reflectometry (OTDR) characterizes optical fibers, by injecting an optical probe pulse into the fiber under test and detecting the backscattered optical signals including Fresnel reflections and Rayleigh backscattering. Increasing pulsewidth of the probe pulse improves the signal to noise ratio (SNR) of the detected signal and accordingly the dynamic range of the OTDR, but degrades the spatial resolution indicating the instrument's ability to resolve two adjacent faults.

In order to overcome this trade-off between the SNR and the resolution, correlation techniques used in wireless radars have been applied to OTDR systems [1], [2]. The correlation techniques have been believed to provide an improved dynamic range without sacrificing the resolution. Periodic pseudorandom codes, however, turned out to be unsuitable for practical systems since they yield a serious dynamic range problem [3].

For a long range application, the complementary correlation OTDR (CC-OTDR) based on the complementary pairs of Golay codes was proposed and experimentally demonstrated [2]. To the best of our knowledge, this is the only existing work which has demonstrated experimental SNR improvement using the correlation technique in a non-coherent OTDR system.
Then, a method using simplex codes was theoretically suggested, which said that the SNR improvement using simplex codes is superior to that obtained using the Golay codes [4]. The main idea of this work was that the SNR of an OTDR can be improved by simultaneous measurements of several distances of the fiber under test, employing simplex codes which has been used to improve the SNR of optical spectrometries [5], [6]. But the idea of this work has not been verified, numerically or experimentally.

In this letter, we propose a practical algorithm and detailed equations to realize the simplex code OTDR. Also we experimentally demonstrate the SNR improvement of OTDR using the simplex codes, by developing an OTDR board which can modulate the probe pulses according to the code sequences. The realized one-way dynamic range improvement over conventional OTDR is shown to be about $4.5 \mathrm{~dB}$, in correspondence with the SNR enhancement formula which was derived in the spectrometry area [6].

\section{Algorithm To REALIZE SIMPLEX CODE OTDR}

The method of measuring optical signals using S-matrix has been widely used in the optical spectrometries for a long time [5], [6]. The S-matrix is a unipolar matrix composed of 1's and 0's, and the rows of this matrix are called simplex codes. It can be derived from a normalized Hadamard matrix which is a bipolar matrix composed of 1 's and -1 's [6]. It has been generally used to detect optical power signals due to its unipolar characteristics, although it provides lower efficiency than the Hadamard matrix. The operation using Hadamard- or S-matrix has been called Hadamard transform, and the fast calculation algorithm, so called Fast Hadamard Transform (FHT), has been developed for the fast operation in a practical system 


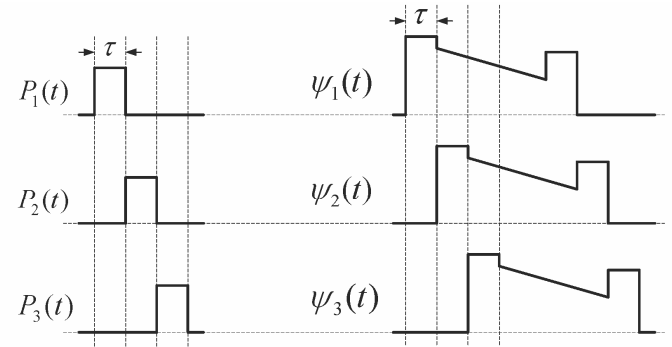

(a)

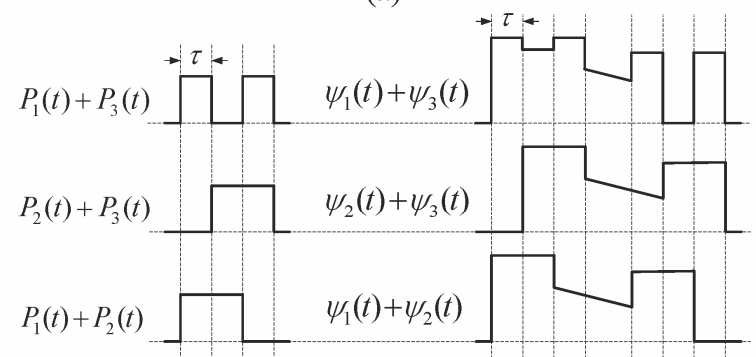

(b)

Fig. 1. Example of the time domain application of the Hadamard transform technique using a S-matrix of order 3.

[7]. The Hadamard transform technique has been applied to the spectrometries by using a mask which consists of holes and blocks spatially [5]. It can be applied to an OTDR system by turning the laser on and off in the time domain, corresponding to 1's and 0's of the S-matrix.

Fig. 1 illustrates our algorithm in case of a S-matrix of order 3. When $\psi_{1}(t)$ represents an OTDR trace to obtain ultimately, it can be measured by launching a single pulse $P_{1}(t)$ into the fiber under test, as shown in the top line of Fig. 1(a). In order to apply the Hadamard transform to the time domain, let us define new pulses $P_{2}(t)=P_{1}(t-\tau)$ and $P_{3}(t)=P_{1}(t-2 \tau)$, where $\tau$ is the pulsewidth of $P_{1}(t)$. Then $\psi_{2}(t)$ and $\psi_{3}(t)$ denote the traces measured by injecting $P_{2}(t)$ and $P_{3}(t)$ into the fiber, respectively. From Fig. 1(a), time relationships among the measured traces as well as among the input pulses are given by

$$
\begin{array}{ll}
P_{2}(t)=P_{1}(t-\tau), & P_{3}(t)=P_{1}(t-2 \tau), \\
\psi_{2}(t)=\psi_{1}(t-\tau), & \psi_{3}(t)=\psi_{1}(t-2 \tau) .
\end{array}
$$

Under these conditions, three coded traces $\eta_{1}(t), \eta_{2}(t)$, and $\eta_{3}(t)$ can be measured by launching simplex code sequences into the fiber, as described in Fig. 1(b) and eq. (2), where $e_{1}(t), e_{2}(t)$, and $e_{3}(t)$ are noise amplitudes added in each measurement at the receiver:

$$
\begin{aligned}
& P_{1}(t)+P_{3}(t) \Rightarrow \eta_{1}(t)=\psi_{1}(t)+\psi_{3}(t)+e_{1}(t) \\
& P_{2}(t)+P_{3}(t) \Rightarrow \eta_{2}(t)=\psi_{2}(t)+\psi_{3}(t)+e_{2}(t) \\
& P_{1}(t)+P_{2}(t) \Rightarrow \eta_{3}(t)=\psi_{1}(t)+\psi_{2}(t)+e_{3}(t)
\end{aligned}
$$

These equations can be represented by Hadamard transform using the S-matrix:

$$
\left(\begin{array}{l}
\eta_{1}(t) \\
\eta_{2}(t) \\
\eta_{3}(t)
\end{array}\right)=S\left(\begin{array}{l}
\psi_{1}(t) \\
\psi_{2}(t) \\
\psi_{3}(t)
\end{array}\right)+\left(\begin{array}{l}
e_{1}(t) \\
e_{2}(t) \\
e_{3}(t)
\end{array}\right), \quad S=\left(\begin{array}{lll}
1 & 0 & 1 \\
0 & 1 & 1 \\
1 & 1 & 0
\end{array}\right)
$$

From Fig. 1(b) and eq. (4), it can be seen that the code length is equal to the order of S-matrix. In order to recover the ultimate OTDR trace $\psi_{1}(t)$, the measured 3 traces are processed by using inverse Hadamard transform as shown in eq. (5), where $\widehat{\psi}_{i}(t)$ represents the estimate of $\psi_{i}(t)$ :

$$
\left(\begin{array}{l}
\widehat{\psi}_{1}(t) \\
\widehat{\psi}_{2}(t) \\
\widehat{\psi}_{3}(t)
\end{array}\right)=S^{-1}\left(\begin{array}{l}
\eta_{1}(t) \\
\eta_{2}(t) \\
\eta_{3}(t)
\end{array}\right)=\frac{1}{2}\left(\begin{array}{ccc}
1 & -1 & 1 \\
-1 & 1 & 1 \\
1 & 1 & -1
\end{array}\right)\left(\begin{array}{l}
\eta_{1}(t) \\
\eta_{2}(t) \\
\eta_{3}(t)
\end{array}\right)
$$

$\widehat{\psi}_{1}(t)=\frac{1}{2}\left\{\eta_{1}(t)-\eta_{2}(t)+\eta_{3}(t)\right\}=\psi_{1}(t)+\frac{e_{1}(t)-e_{2}(t)+e_{3}(t)}{2}$

$\widehat{\psi}_{2}(t)=\frac{1}{2}\left\{-\eta_{1}(t)+\eta_{2}(t)+\eta_{3}(t)\right\}=\psi_{2}(t)+\frac{-e_{1}(t)+e_{2}(t)+e_{3}(t)}{2}$

$\widehat{\psi}_{3}(t)=\frac{1}{2}\left\{\eta_{1}(t)+\eta_{2}(t)-\eta_{3}(t)\right\}=\psi_{3}(t)+\frac{e_{1}(t)+e_{2}(t)-e_{3}(t)}{2}$

From averaging these estimates after time-shifting them by multiples of $\tau$, we can finally obtain the restored trace

$$
\begin{aligned}
& \frac{\widehat{\psi}_{1}(t)+\widehat{\psi}_{2}(t+\tau)+\widehat{\psi}_{3}(t+2 \tau)}{3}=\psi_{1}(t)+\frac{e_{1}(t)-e_{2}(t)+e_{3}(t)}{6}+\quad(6) \\
& \frac{-e_{1}(t+\tau)+e_{2}(t+\tau)+e_{3}(t+\tau)+e_{1}(t+2 \tau)+e_{2}(t+2 \tau)-e_{3}(t+2 \tau)}{6},
\end{aligned}
$$

using the time relationships in eq. (1) additionally. Therefore, the mean square error (MSE) in the finally restored trace becomes

$E\left\{\left(\frac{\widehat{\psi}_{1}(t)+\widehat{\psi}_{2}(t+\tau)+\widehat{\psi}_{3}(t+2 \tau)}{3}-\psi_{1}(t)\right)^{2}\right\}=\frac{9 \sigma^{2}}{36}=\frac{1}{4} \sigma^{2}$,

$\because E\left\{e_{i}(t)\right\}=0, E\left\{e_{i}^{2}(t)\right\}=\sigma^{2}, E\left\{e_{i}(t) e_{j}(t)\right\}=0(i \neq j)$

under the assumption that the noise $e_{i}(t)$ 's are uncorrelated, zero-mean random processes with variance $\sigma^{2}$. Since the restored trace in eq. (6) was obtained from 3 traces measured by 3 probe shots, we should calculate the SNR enhancement over conventional OTDR, namely coding gain, by comparing with a result obtained from $\mathrm{N}=3$ conventional averaging. The MSE of $\mathrm{N}=3$ averaging is $\sigma^{2} / 3$, so the coding gain at the code length 3 is given by

$$
\sqrt{\frac{\sigma^{2}}{3} / \frac{\sigma^{2}}{4}}=\frac{2}{\sqrt{3}} \text {. }
$$


The described time domain algorithm can be easily extended to the general case of code length $L$, while the above procedure depicts the specific case of code length 3 . Then the coding gain for the general code length $L$ becomes

$$
\frac{L+1}{2 \sqrt{L}},
$$

which is the same as that derived in the Hadamard transform spectrometries [6].

\section{NOISE ANALYSIS FOR BAND-LIMITED RECEIVERS}

In eq. (7), we assumed that the noise samples in the same noise ensemble are uncorrelated, i.e., $E\left\{e_{N}(t) e_{N}(t+\tau)\right\}=0,(\tau \neq 0)$, as well as that the noise in the receiver is i.i.d., w.w.s. (independent, identically distributed, wide-sense stationary) random process. The assumption that auto-correlation values are zero, however, is valid only when the receiver has an infinite bandwidth. Therefore, with a real band-limited receiver, we should consider the non-zero auto-correlation value, i.e., $E\left\{e_{N}(t) e_{N}(t+\tau)\right\}=R_{N}(\tau) \neq 0,(\tau \neq 0)$ to calculate the exact noise power in the final trace (eq. (6)). This is because the Hadamard transform in the simplex code OTDR is performed in the time domain, not in the space domain as in the spectrometries.

Now we derive decoding equations for the general $L$-bit codes and the new coding gain formula which depends on the receiver bandwidth and the probe pulsewidth, as well as the code length. When we represent a S-matrix of order $L$ as $S_{L}$ and its inverse matrix as $\boldsymbol{S}_{L}^{-1}$ respectively, trace estimates are obtained as eq. (10) by performing the inverse Hadamard transform to the measured traces .

$$
\left(\begin{array}{c}
\widehat{\psi}_{1}(t) \\
\widehat{\psi}_{2}(t) \\
\vdots \\
\hat{\psi}_{L}(t)
\end{array}\right)=S_{L}{ }^{-1}\left(\begin{array}{c}
\eta_{1}(t) \\
\eta_{2}(t) \\
\vdots \\
\eta_{L}(t)
\end{array}\right)=\left(\begin{array}{c}
\psi_{1}(t) \\
\psi_{2}(t) \\
\vdots \\
\psi_{L}(t)
\end{array}\right)+S_{L}^{-1}\left(\begin{array}{c}
e_{1}(t) \\
e_{2}(t) \\
\vdots \\
e_{L}(t)
\end{array}\right)
$$

By inversely time-shifting each row in eq. (10) by multiples of $\tau$, and introducing matrix $\boldsymbol{T}_{L}$ which is the normalized matrix of $S_{L}$, eq. (10) can be developed as:

$$
\begin{gathered}
\left(\begin{array}{c}
\widehat{\psi}_{1}(t) \\
\widehat{\psi}_{2}(t+\tau) \\
\vdots \\
\widehat{\psi}_{L}(t+(L-1) \tau)
\end{array}\right)=\left(\begin{array}{c}
\psi_{1}(t) \\
\psi_{2}(t+\tau) \\
\vdots \\
\psi_{L}(t+(L-1) \tau)
\end{array}\right)+\frac{2}{L+1} T_{L}\left(\begin{array}{c}
e_{1}(t) \\
e_{2}(t+\tau) \\
\vdots \\
e_{L}(t+(L-1) \tau)
\end{array}\right) \\
T_{L}=\frac{L+1}{2} S_{L}^{-1}=\left(\begin{array}{cccc}
T_{1,1} & T_{1,2} & \cdots & T_{1, L} \\
T_{2,1} & T_{2,2} & \cdots & \vdots \\
\vdots & \vdots & \vdots & \vdots \\
T_{L, 1} & \cdots & \cdots & T_{L, L}
\end{array}\right), \quad T_{j, k} \in\{1,-1\}
\end{gathered}
$$

Using the following relation extended from eq. (1),

$$
\psi_{i}(t+(i-1) \tau)=\psi_{1}(t),(i=1,2, \cdots, L)
$$

$L$ equations can be developed from eq. (11) as follows:

$$
\begin{aligned}
& \widehat{\psi}_{1}(t)=\psi_{1}(t)+\frac{2}{L+1} \sum_{k=1}^{L} T_{1, k} e_{k}(t) \\
& \widehat{\psi}_{2}(t+\tau)=\psi_{1}(t)+\frac{2}{L+1} \sum_{k=1}^{L} T_{2, k} e_{k}(t+\tau) \\
& \vdots \\
& \widehat{\psi}_{L}(t+(L-1) \tau)=\psi_{1}(t)+\frac{2}{L+1} \sum_{k=1}^{L} T_{L, k} e_{k}(t+(L-1) \tau)
\end{aligned}
$$

Then, by adding all terms in each side and dividing them by $L$, we can achieve the following equation for the final trace, together with the exact noise terms:

$\frac{1}{L} \sum_{k=1}^{L} \widehat{\psi}_{k}(t+(k-1) \tau)=\psi_{1}(t)+\frac{2}{L(L+1)} \sum_{j=1}^{L} \sum_{k=1}^{L} T_{j, k} e_{k}(t+(L-1) \tau)$

Therefore, the mean square error (MSE) in the finally restored trace can be written as

$$
\begin{aligned}
& E\left\{\left(\frac{1}{L} \sum_{k=1}^{L} \widehat{\psi}_{k}(t+(k-1) \tau)-\psi_{1}(t)\right)^{2}\right\} \\
= & \frac{4}{L^{2}(L+1)^{2}} E\left\{\left(\sum_{j=1}^{L} \sum_{k=1}^{L} T_{j, k} e_{k}(t+(L-1) \tau)\right)^{2}\right\} \\
= & \frac{4}{L^{2}(L+1)^{2}}\left\{L^{2} \sigma^{2}-\sum_{i=1}^{L-1}(L-i) R_{N}(i \tau)\right\} \\
= & \frac{4}{(L+1)^{2}}\left\{\sigma^{2}-\frac{1}{L^{2}} \sum_{i=1}^{L-1}(L-i) R_{N}(i \tau)\right\},
\end{aligned}
$$

where we assumed that the noise in the receiver is zero mean, i.i.d., w.w.s. random process, and utilized the fact that total sum of each row in $\boldsymbol{T}_{\boldsymbol{L}}$ is always -1 , as follows:

$E\left\{e_{i}(t+\zeta)\right\}=0, E\left\{e_{i}^{2}(t+\zeta)\right\}=\sigma^{2}$,

$E\left\{e_{i}(t) e_{i}(t+\zeta)\right\}=R_{i}(\zeta)=R_{N}(\zeta),(i=1,2, \cdots, L)$

$E\left\{e_{i}(t) e_{j}(t+\zeta)\right\}=0(i \neq j), \sum_{k=1}^{L} T_{j, k}=-1(j=1,2, \cdots, L)$

Since the final trace in eq. (14) is obtained from $L$ measurement, the coding gain over conventional OTDR is calculated as eq. (17), by comparison with $\mathrm{N}=L$ conventional averaging.

$$
\begin{aligned}
& \sqrt{\frac{\sigma^{2}}{L}} / \sqrt{\frac{4}{(L+1)^{2}}\left\{\sigma^{2}-\frac{1}{L^{2}} \sum_{i=1}^{L-1}(L-i) R_{N}(i \tau)\right\}} \\
& =\frac{L+1}{2 \sqrt{L}} / \sqrt{1-\frac{1}{L^{2} \sigma^{2}} \sum_{i=1}^{L-1}(L-i) R_{N}(i \tau)}
\end{aligned}
$$




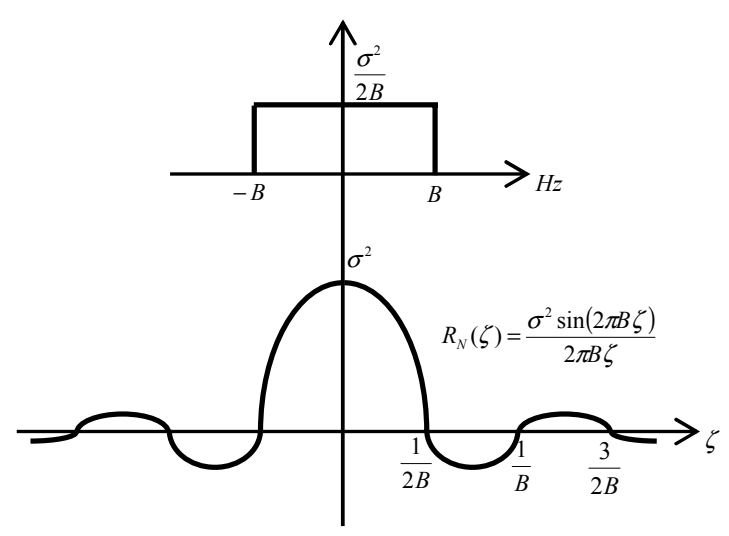

Fig. 2. Frequency response of the ideal band-limited receiver and the auto-correlation function of the noise from the receiver.

From eq. (17) we can see that the SNR of the finally restored trace varies with the auto-correlation function of the noise in the band-limited receiver and the probe pulsewidth. For the simple example, if we assume the frequency response of the band-limited receiver to be an ideal low-pass filter with bandwidth $B \mathrm{~Hz}$ as shown in fig. (2), the coding gain becomes

$$
\frac{L+1}{2 \sqrt{L}} / \sqrt{1-\frac{1}{L^{2}} \sum_{i=1}^{L-1}(L-i) \frac{\sin (2 i \pi B \tau)}{2 i \pi B \tau}} .
$$

Therefore, the SNR enhancement is the greatest when

$$
\sum_{i=1}^{L-1}(L-i) \frac{\sin (2 i \pi B \tau)}{2 i \pi B \tau}
$$

has the maximum value, and vice versa. From fig. (2) and eq. (19), we can summarize this by the relationship between the receiver bandwidth $B$ and the probe pulsewidth $\tau$. The additional SNR variation due to the band-limited characteristics degrades the coding gain the most when

$$
\tau=\frac{3}{4 B}
$$

improves it slightly when

$$
\frac{k}{B} \leq \tau \leq \frac{2 k+1}{2 B},(k=0,1,2, \cdots)
$$

improves it the most when

$$
(n-1) \tau<\frac{1}{2 B} .
$$

Although this analysis corresponds to the ideal receiver with bandwidth $B$, more accurate result can be obtained by using the exact frequency response of the real receiver, and it is expected that the conditions will be derived similar to those derived in the ideal receiver case.

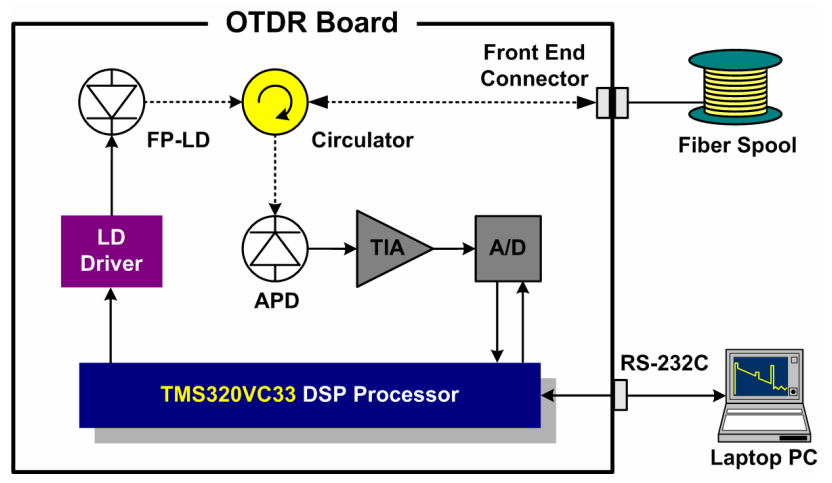

Fig. 3. Experimental setup.

\section{EXPERIMENTAL VERIFICATION}

To verify our algorithm and realizing equations, we developed an OTDR board which is capable of modulating the probe pulses according to the given code sequences (simplex codes), sampling the measured trace, and transmitting the sampled data to a PC. The measured traces are transmitted to the PC using RS-232C, and the final trace is restored and displayed at the PC. Fig. 3 shows a block diagram of the experimental arrangement. Texas Instrument's TMS320VC33 was selected for a digital signal processor (DSP) which takes charge of pulse coding, trace acquisition and averaging, command and data communications, and so on. A Fabry-Perot laser diode (FP-LD) and an avalanche photodiode (APD) were used for the optical source and detector, respectively. For a specific experiment, two fiber spools of $20 \mathrm{~km}$ SMF and a laptop PC were connected to the board. The spools were connected using $\mathrm{FC} / \mathrm{PC}$ connectors, while the end of the link was tailed with a FC/APC connector. From this setup, Fig. 4(a) shows an averaged trace from 255 probe shots using the conventional single pulse method. The pulsewidth of the probe pulse is $500 \mathrm{~ns}$, yielding a spatial resolution of about 50m. Fig. 4(b) represents a trace restored from 255 measured traces using the simplex coding technique. It can be observed that the SNR of the trace in Fig. 4(b) is much better than that in Fig. 4(a), while the measurement conditions, such as the laser peak power, the measurement time, and the resolution, are the same in the two cases. The overhead time spent in the trace restoration can be ignored compared to the measurement time, when the fast Hadamard transform (FHT) is used. In Fig. 4(b), the one-way dynamic range was enhanced by about $4.5 \mathrm{~dB}$ compared to Fig. 4(a), in correspondence with the coding gain of $9 \mathrm{~dB}$ which can be predicted by substituting 255 for $L$ in eq. (9). 

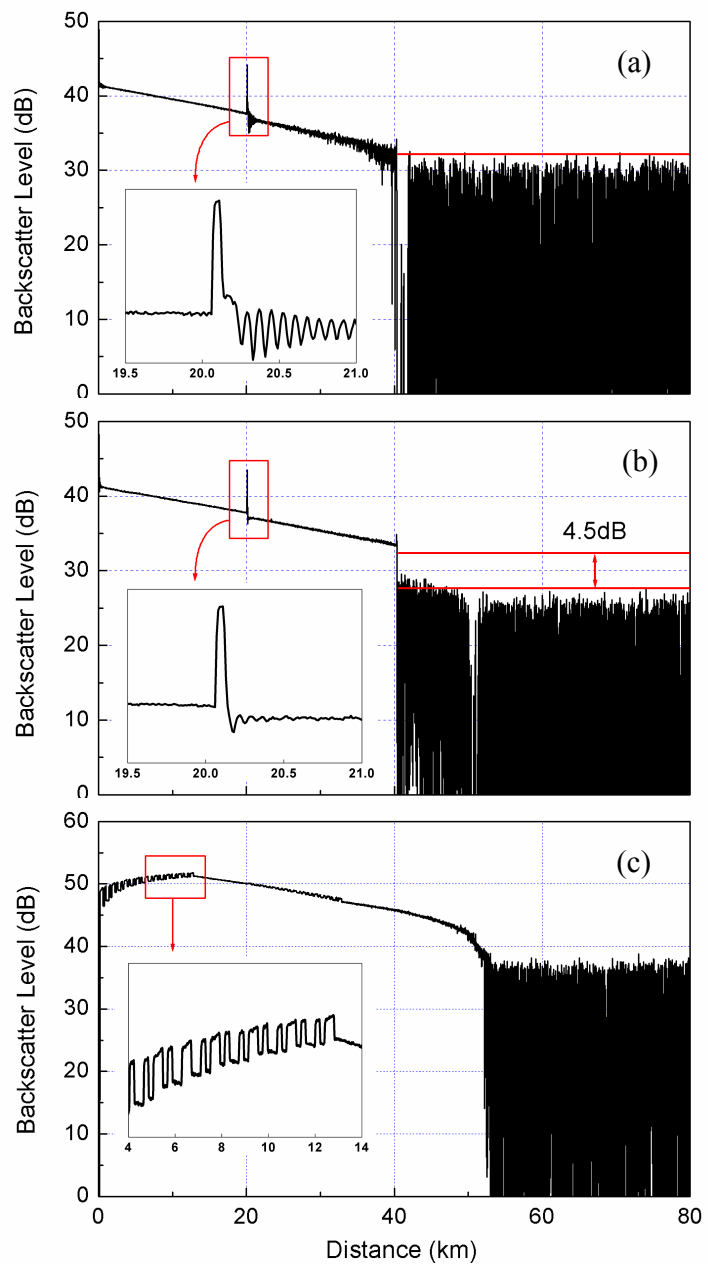

Fig. 4. (a) OTDR trace using conventional single pulse method, (b) Finally restored trace from 255 coded traces measured with simplex codes, (c) One of the 255 coded traces.

\section{CONCLUSION}

We demonstrated SNR improvement of OTDR using simplex codes by experiment, for the first time. For this, we proposed a practical algorithm and detailed equations to realize the simplex code OTDR. Also we experimentally demonstrated the SNR improvement of OTDR, using the OTDR board to which the proposed realizing algorithm and equations are applied. The experimental results showed excellent agreement with theory.

\section{REFERENCES}

[1] K. Okada and K. Hashimoto, "Optical cable fault location using correlation technique", Electronics Lett., Vol. 16, pp. 629-630, 1980.

[2] M. Nazarathy, S. A. Newton, R. P. Giffard, D. S. Moberly, F. Sischka, W. R. Trutna, Jr., and S. Foster, "Real-time long range complementary correlation optical time domain reflectometer," J. Lightwave Technol., vol. 7, pp. 24-38, Jan. 1989

[3] P. Healey, "Review of long wavelength single-mode optical fiber reflectometry techniques," J. Lightwave Technol., vol. LT-3, pp. 876-886, Aug. 1985.

[4] M. Jones, "Using simplex codes to improve OTDR sensitivity," IEEE Photon. Technol. Lett., vol. 15, pp. 822824, Jul. 1993.

[5] J. A. Decker, "Experimental realization of the multiplex advantage with a Hadamard-transform spectrometer," Applied Optics, vol. 10, pp. 510-514, 1971.

[6] M. Harwit and N. J. Sloane, Hadamard transform optics, New York: Academic, 1979.

[7] E. E. Fenimore and G. S. Weston, "Fast delta Hadamard transform," Applied Optics, vol. 20, pp. 3058-3067, 1981. 